\section{S78 DIFFERENTIATION OF MONOCYTES TO PRO- INFLAMMATORY FORMS IS INFLUENCED BY CIGARETTE SMOKE AND HLA TYPE IN COPD}

doi:10.1136/thoraxjnl-2011-201054b.78

P R Newby, C Schmutz, C R Buckley, R A Stockley, A M Wood. University of Birmingham, Birmingham, UK

Background There are many genetic influences documented on both lung function and susceptibility to COPD. In GWAS of pulmonary function several hits in the region of the MHC on chromosome 6 have been found, and we have shown previously that HLADR3 positive individuals have lower $\mathrm{FEV}_{1}$ than those without this HLA type. This is an HLA type classically associated with autoimmunity. Interactions between HLA type and cigarette smoke are recognised in autoimmune diseases.

Hypothesis HLA type influences differentiation of monocytes in the presence of cigarette smoke.

Methods 15 ex and never smokers with COPD and 5 healthy controls were studied. PBMCs were isolated and exposed to varying concentrations of cigarette smoke extract (CSE) for $90 \mathrm{~min}$. CD14 and CD16 markers were used in flow cytometry to ascertain relative expression and absolute cell counts for each monocyte subpopulation, defined as CD14++CD16- (classical), CD14++CD16+ (anti-inflammatory) and $\mathrm{CD} 14+\mathrm{CD} 16++$ (non-classical). Within the patient group differences in baseline profile and response to CSE were compared between ex-smokers and those that had never smoked. Patients were HLA class II typed as described previously ${ }^{1}$ and the same comparisons made between DR3 positive and negative patients.

Results At baseline the MFI for CD14 was lower in COPD than health $(p=0.04)$, although no clear differences in cell counts were seen. Counts were generally higher in ex-smokers, although no clear differences in subpopulations were seen. On exposure to cigarette smoke there was a dose dependent rise in classical monocytes, which was more marked in DR3+ patients and never smokers.

Conclusions CSE induces a pro-inflammatory phenotype of monocytes, and this occurs most in HLADR3 + individuals. This could be the mechanism behind lower $\mathrm{FEV}_{1}$ in $\mathrm{DR}_{3}+$ individuals.

\section{REFERENCE}

1. Bunce M, et al. Tissue antigens. 1995;46:355-67.

\section{Severe asthma in children and adults S79 SPECIFIC FUNCTIONAL ANTIBODY DEFICIENCY IS ASSOCIATED WITH A REDUCTION LUNG FUNCTION IN THE SEVERE ASTHMA POPULATION}

doi:10.1136/thoraxjnl-2011-201054b.79

H Wardman, R Niven, G McCumesky, L J Holmes, D Menzies, G Tavernier. University Hospital of South Manchester, Manchester, UK

Background Haemophilus influenzae type B (Hib) and Streptoccus pneumoniae are leading causes of LRTI in the severe asthma population. Patients attending our regional severe asthma service have functional antibody levels tested against these two bacteria. Those with weakened immune systems may go on to require immunoglobulin therapy. The prevalence and impact of specific antibody deficiency within the severe asthma population has yet to be established.

Objective We sought to quantify the number of patients who are deficient in antibodies against Hib and $S$ pneumoniae within the severe asthma population, both at initial assessment and after vaccination.

Methods Data from our regional clinic stored on a National Severe Asthma database was supplemented with information from the UHSM clinical results database, which contains blood antibody levels against $H i b$ and $S$ pneumoniae and lung physiology. Deficiency in Hib was defined as antibody levels of $<0.15 \mu \mathrm{g} / \mathrm{ml}$ and for $S$ pneumoniae, 6 out of 12 strains tested measuring $>0.35 \mu \mathrm{g} / \mathrm{ml}$. Only patients assessed after January 2008 were included. The prevalence of radiological abnormality, Aspergillus sensitisation, blood and sputum eosinophil counts, and lung function between severe asthma patients with antibody deficiency, and those with normal antibody levels were then compared.

Results Among the patients tested for immunity to $S$ pneumoniae $(\mathrm{n}=94)$ and $\mathrm{Hib}(\mathrm{n}=97), 33 \%$ and $51 \%$ respectively were found to be deficient at initial assessment. In patients with baseline immune deficiency, $70 \%$ of those that received the Pneumovax vaccine had persistently low antibody levels against $S$ pneumoniae, $(p=0.03)$, and $20 \%$ who received Menitorix remained deficient in Hib antibodies. The mean post bronchodilator $\mathrm{FEV}_{1}$ for patients with normal $S$ pneumoniae antibody levels was $82.4 \%$ predicted compared to $68.8 \%$ predicted in those who were deficient at initial assessment $(p=0.018)$, and $65.3 \%$ predicted in patients with persistently low antibody levels even after vaccination $(p=0.049)$. All other variables showed no difference between the groups.

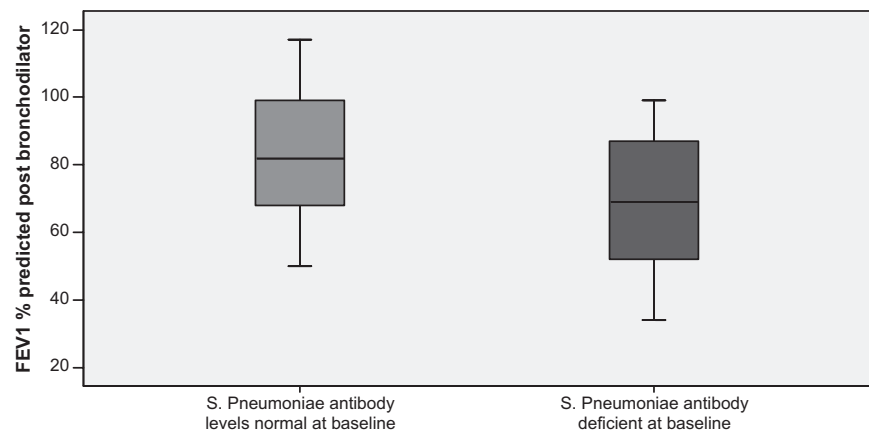

Abstract S79 Figure 1

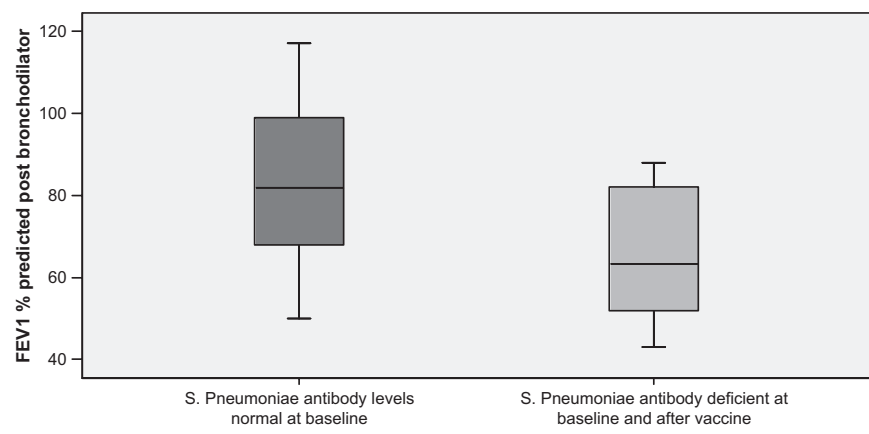

Abstract S79 Figure 2

Conclusion This study demonstrates for the first time that antibody deficiency to $S$ pneumoniae and $H i b$ is common in patients with severe asthma, can persist despite vaccination in a significant proportion of individuals and is associated with worse lung function.

\section{S80 LOCALISATION OF THE SITE OF FIXED AIRFLOW OBSTRUCTION IN MODERATE TO SEVERE ASTHMA USING HYPERPOLARISED HELIUM-3 MRI}

doi:10.1136/thoraxjnl-2011-201054b.80

${ }^{1} \mathrm{~S}$ Gonem, ${ }^{2} \mathrm{~B}$ Ball, ${ }^{1} \mathrm{~S}$ Corkill, ${ }^{1} \mathrm{D}$ Desai, ${ }^{1} \mathrm{~A}$ Singapuri, ${ }^{3} \mathrm{P}$ Gustafsson, ${ }^{2} \mathrm{~J}$ Owers-Bradley, ${ }^{1} \mathrm{C}$ Brightling, ${ }^{1} \mathrm{~S}$ Siddiqui. ${ }^{1}$ Glenfield Hospital, Leicester, UK; ${ }^{2}$ University of Nottingham, Nottingham, UK; ${ }^{3}$ Queen Silvia Children's Hospital, Göteborg, Sweden

Introduction and Objectives Moderate and severe asthma are often associated with a degree of fixed airflow obstruction. We aimed to 